\title{
CARACTERIZAÇÃO DO PROCESSAMENTO AUDITIVO DAS CRIANÇAS COM DISTÚRBIO DE LEITURA E ESCRITA DE 8 A 12 ANOS EM TRATAMENTO NO CENTRO CLÍNICO DE FONOAUDIOLOGIA DA PONTIFÍCIA UNIVERSIDADE CATÓLICA DE MINAS GERAIS
}

\author{
Characterization of auditive processing of children with \\ of reading and writing disturbance from 8 to 12 year old \\ in treatment at the Clinical Center of Speech and Hearing \\ of Pontifical University Catholic of Minas Gerais
}

\begin{abstract}
Cíntia Santos Silva Machado ${ }^{(1)}$, Hálida Larissa Batista da Silva do Valle ${ }^{(2)}$, Karen Maria de Paula ${ }^{(3)}$, Sheilla da Silva Lima ${ }^{(4)}$
\end{abstract}

\begin{abstract}
RESUMO
Objetivo: verificar possíveis desordens do processamento auditivo em crianças com distúrbio de leitura e escrita. Método: foram selecionadas 15 crianças, com audição normal, na faixa etária de 8 a 12 anos, com baixo rendimento escolar e que se encontravam em tratamento no setor de linguagem escrita no Centro Clínico de Fonoaudiologia da Pontifícia Universidade Católica de Minas Gerais. Os indivíduos foram submetidos aos testes: Pediatric Speech Intelligibility test, - Staggered Spondaic Word test, Fala com Ruído, Fala Filtrada, Gap in Noise e Padrão de Duração e Freqüência Melódicos. Resultados: verificou-se que todos os indivíduos apresentaram alterações em pelo menos uma habilidade auditiva do processamento auditivo.Conclusão: Verificou-se estreita e estrita relação entre as habilidades do processamento auditivo e o distúrbio de leitura e escrita. Os procedimentos que se destacaram na identificação de anormalidades no processamento neurológico da informação auditiva foram os testes SSW, PSI com palavras no ruído e Teste de Padrão de Freqüência melódico.
\end{abstract}

Descritores: Linguagem; Baixo Rendimento Escolar; Audição; Criança; Leitura

(1) Fonoaudióloga; Docente do curso de Fonoaudiologia da Pontifícia Universidade Católica de Minas Gerais; Mestre em Ciências da Saúde da Criança e do Adolescente pela Faculdade de Medicina da Universidade Federal de Minas Gerais.

(2) Fonoaudióloga; Pós-graduada em Gestão em Fonoaudiologia pela Faculdade de Estudos Administrativos de Minas Gerais.

(3) Fonoaudióloga; Mestranda em Estudos Lingüísticos pela Universidade Federal de Minas Gerais.

(4) Fonoaudióloga pela Pontifícia Universidade Católica de Minas Gerais.

Conflito de interesses: inexistente

\section{INTRODUÇÃO}

Os transtornos de aprendizagem têm sido foco de inúmeras pesquisas durante os últimos anos. Tal interesse por parte de diversas áreas da ciência deve-se à importância da aprendizagem para o completo desenvolvimento sócio-cultural e até mesmo emocional do ser humano ${ }^{1}$.

É crescente o número de crianças, dentro do ciclo do fracasso escolar, que apresentam dificuldade no aprendizado da leitura e da escrita e que permanecem em situação de sala de aula sem 
acompanhar as referidas atividades. Assim, o fracasso escolar ainda é um dos grandes problemas da nossa sociedade e propostas para minimizar tal problema devem ser desenvolvidas².

Dentre as diversas nomenclaturas e definições para os problemas específicos de aprendizagem pode-se citar: disortografia e disgrafia para problemas específicos de escrita, dislexia para problemas de leitura, distúrbio da aprendizagem, dificuldade específica de aprendizagem e dificuldade de leitura ${ }^{3}$.

Também é válido ressaltar que, há uma vasta gama de terminologias para denominar os distúrbios de leitura e de escrita, dentre os quais podemos citar: dislexia, dislexia específica de desenvolvimento, dificuldade específica de leitura, distúrbio específico de leitura, atraso específico de leitura, leitores fracos, distúrbios de leitura e distúrbio do aprendizado da linguagem ${ }^{4}$. Portanto, no presente estudo será adotado o termo "distúrbios de leitura e escrita" como um termo que denomina uma categoria ampla de dificuldades nessas habilidades, uma vez que tanto na literatura nacional como na internacional, todas as denominações, anteriormente citadas, são comumente utilizados como sendo equivalentes ${ }^{4-7}$.

O distúrbio de leitura e escrita é uma manifestação referente ao desenvolvimento da linguagem, que tem por característica a dificuldade na aquisição e/ou no desenvolvimento da linguagem escrita por indivíduos que apresentam déficits tanto de decodificação fonológica como de compreensão da linguagem oral e/ou escrita. Ainda que a manifestação seja mais evidente durante o aprendizado da leitura e da escrita, alguns sinais de dificuldades mais amplas de linguagem como vocabulário pobre, uso inadequado da gramática e problemas no processamento fonológico podem aparecer desde os anos pré-escolares. Nas séries iniciais da escolaridade, além de dificuldades em reconhecer palavras e compreender a leitura, podem apresentar problemas de compreensão auditiva e de discurso ${ }^{3,8}$.

Os processos da linguagem e da aprendizagem são bastante complexos, envolvem redes de neurônios distribuídas em diferentes regiões cerebrais e se relacionam à percepção da fala, sendo dependentes da integridade auditiva periférica e central ${ }^{8}$.

Embora existam diferentes causas para os distúrbios de aprendizagem, oriundas de processos patológicos diversos, estes podem, também, acometer indivíduos aparentemente saudáveis, como visto em alguns estudos sobre a neuropsicologia da aprendizagem, nos quais essas alterações podem estar presentes em indivíduos com total integridade orgânica e podem manifestar-se de diferentes formas, como por exemplo; dificuldades de atenção, memorização, desinteresse, compreensão, problemas de comportamento ${ }^{3}$.

Entende-se por processamento auditivo (PA) o conjunto de habilidades específicas das quais o indivíduo depende para interpretar o que ouve. Quando o indivíduo perde parcialmente ou totalmente a função da análise das imagens auditivas, estamos diante de um distúrbio do processamento auditivo ${ }^{9}$.

O questionamento de uma possível relação entre as desordens do processamento auditivo/ distúrbio de leitura e escrita, se faz presente, visto que, o processamento auditivo refere-se ao conjunto de habilidades necessárias à interpretação do que se ouve, tais como: detecção, sensação, discriminação, localização, reconhecimento, compreensão, atenção e memória e o déficit em tais habilidades poderão estar presentes nas crianças com queixa de dificuldade no aprendizado escolar ${ }^{10,11}$.

É cada vez mais evidente em clínicas fonoaudiológicas a presença de crianças com distúrbios do processamento auditivo em associação com os distúrbios de linguagem oral ${ }^{1}$ e/ou escrita ${ }^{2,3}$. Neste contexto, há a necessidade emergente de estudar diferentes técnicas de reabilitação auditiva que possam ser mais eficazes no tratamento destas habilidades auditivas e de linguagem, constituindo esta uma das vertentes de pesquisas na área do processamento auditivo ${ }^{12}$.

O papel do processamento auditivo em problemas de aprendizagem adquiriu notoriedade nos últimos anos. A partir desta perspectiva e, salientando o fato de que a repetência é uma forma de exclusão social, o presente estudo tem por objetivo avaliar se as desordens do processamento auditivo contribuem de alguma maneira, no processo de distúrbio de leitura e escrita levando, consequentemente à repetência, ou retenção escolar.

As habilidades do processamento auditivo são mediadas pelas estruturas do tronco encefálico e córtex cerebral, permitindo analisar e interpretar os estímulos sonoros (localizar a fonte sonora, memorizar sons verbais, etc.), o que é fundamental no desenvolvimento da fala e da linguagem ${ }^{13,14}$.

O processamento auditivo é desenvolvido desde os primeiros anos de vida, e as habilidades perceptuais auditivas envolvidas neste processamento são: atenção seletiva - habilidade em focar um determinado estímulo sonoro em meio a outros sons competitivos, auditivos ou visuais; detecção do som - habilidade de identificação da presença do som; sensação sonora - é o nome dado quando um estímulo sonoro é recebido via sentido da audição; discriminação - habilidade de detectar diferenças entre os padrões de estímulos sonoros; 
localização sonora - habilidade em identificar o local de origem do som; reconhecimento - habilidade em identificar corretamente um evento sonoro previamente reconhecido; compreensão - habilidade de compreender/deduzir o significado da informação auditiva; memória - habilidade que permite armazenar, arquivar informações acústicas para poder recuperá-las depois ${ }^{13,15}$.

As desordens do processamento auditivo têm como prováveis causas, alterações neurológicas ou alterações sensoriais auditivas - as perdas auditivas neurossensoriais e condutivas, mesmo as transitórias, decorrentes de episódios de otite média na infância que, dentre as afecções otológicas, é a mais freqüente. As disfunções centrais podem ocorrer por disfunção neuromorfológica, atraso de maturação do sistema nervoso auditivo central e distúrbios, doenças ou lesões neurológicas e otológicas ${ }^{15}$.

A avaliação do processamento auditivo surgiu como um meio de esclarecer manifestações auditivas que os testes convencionais não conseguiam explicar. Na maioria das vezes, os resultados desses testes convencionais são compatíveis com a normalidade, no entanto, permanecem ainda queixas quanto ao uso funcional e eficiente da audição ${ }^{15}$.

Algumas desordens podem acometer o processamento auditivo levando o indivíduo a uma dificuldade em lidar com as informações que the chegam pela audição embora seja diferente de surdez. Dentre as características mais comuns associadas a crianças com distúrbio do processamento auditivo estão déficits de compreensão da fala na presença de ruído, distração, atenção reduzida, dificuldade de comunicação, baixo desempenho acadêmico, memória auditiva limitada, dificuldade de identificação da idéia principal de um enunciado, baixa capacidade de interpretação de palavras, frases, anedotas, metáforas, trocadilhos e analogias de sentido ambíguo, alteração na emissão verbal e dificuldade de resgate verbal ${ }^{16}$.

Acredita-se que a avaliação do processamento auditivo em escolares com queixa de aprendizagem pode contribuir em muito para um diagnóstico mais preciso e para uma melhor orientação terapêutica ${ }^{13}$.

A proposta desta pesquisa é destacar a importância de uma intervenção precoce em crianças portadoras de distúrbio de leitura e escrita, por parte de diversos profissionais, sejam eles professores, fonoaudiólogos, médicos, dentre outros, objetivando um diagnóstico precoce e um tratamento adequado para essas crianças, visto que, por trás do mau desempenho ou evasão escolar, sempre há fortes indícios de dificuldades de aprendizagem relacionadas à linguagem podendo estas, estarem ligadas às desordens no processamento auditivo ${ }^{17}$.

Assim, o objetivo deste estudo é verificar possíveis desordens do processamento auditivo em crianças com distúrbio de leitura e escrita.

\section{MÉTODO}

Foram selecionados para participar deste estudo, todos os pacientes, de ambos os gêneros, em atendimento no ambulatório de Linguagem Escrita do Centro Clínico de Fonoaudiologia da Pontifícia Universidade Católica de Minas Gerais. Os prontuários desses pacientes, nos quais constavam exames audiológicos previamente realizados, foram analisados com o objetivo de caracterizar a população estudada e de avaliar se os participantes respeitavam os critérios de inclusão estabelecidos para este estudo, ou seja, foram excluídos da pesquisa os indivíduos que não tinham idade entre 8 e 12 anos e que não apresentavam audição dentro dos limites de normalidade segundo critério de classificação do grau da perda auditiva ${ }^{18}$. Sendo assim, participaram deste estudo 15 sujeitos.

Os responsáveis pelos sujeitos responderam a um questionário, por meio do qual foram levantados dados sobre: suas habilidades e/ou dificuldades, comportamento emocional, relações sociais, histórico de doenças, desenvolvimento de fala e linguagem e história escolar. Os responsáveis pelos participantes da pesquisa assinaram também o Termo de Consentimento, por meio do qual foram esclarecidos sobre os objetivos do estudo.

Antes da avaliação do processamento auditivo realizou-se meatoscopia (observação do conduto auditivo) realizada por meio do aparelho otoscópico Welch Allyn com o objetivo de verificar a ausência de corpos no meato acústico externo.

Posteriormente, os indivíduos foram submetidos aos seguintes testes do processamento auditivo ${ }^{15}$ : Teste de Fala Filtrada, para o qual foi utilizado o CD volume 1 , faixa 2 , que avalia a habilidade auditiva de fechamento por meio do mecanismo fisiológico da reconhecimento de sons fisicamente distorcidos; SSW (Staggered Spondaic Word test), usando o CD volume 2 , faixa 6 , que avalia a habilidade de figura-fundo por meio do mecanismo fisiológico de reconhecimento de sons em escuta dicotica; PSI (Pediatric Speech Intelligibility test) com palavras - monossílabos com figuras, que avalia se o indivíduo é capaz de selecionar a mensagem principal de sons competitivos (figura-fundo), e foi utilizado o CD volume1, faixa 4; Fala com ruído Branco, que avalia a habilidade de fechamento auditivo, contido no CD volume 1, faixa 2, compara os acertos obtidos na presença de ruído com aqueles obtidos 
na ausência de ruídos ${ }^{15}$. O teste PSI com palavras é um teste que avalia as habilidades auditivas de figura-fundo para palavras (figuras/história), que é a capacidade do indivíduo de identificar a mensagem primária na presença de sons competitivos, e o fechamento (monossílabos/ruído) que é a habilidade de perceber o todo (palavra ou mensagem) quando partes são omitidas.

O teste Gap In Noise (GIN) ${ }^{19}$ gravado em CD, com intensidade de $50 \mathrm{~dB}$ NS (de acordo com média tritonal) e a condição de aplicação do teste foi sempre monoaural ${ }^{19}$; e a habilidade auditiva avaliada é a resolução temporal. O teste de Padrão de Duração e de Freqüência Melódicos (Taborga) gravado em CD com estímulo provido por uma flauta, compostos por seqüências de 4 tons com 10 itens cada, no qual avalia a habilidade auditiva de ordenação temporal e discriminação de sons quanto aos aspectos de freqüência e duração, por meio do mecanismo fisiológico de ordenação temporal de sons não-verbais ${ }^{20}$. Ambos os testes avaliam o processamento temporal.

Os equipamentos usados para realizar os procedimentos selecionados foram o audiômetro de dois canais Midimate 622, cabina acústica , e um aparelho para tocar $C D$, marca Sony, e os $C D s$ contendo a gravação dos estímulos para a aplicação dos testes auditivos.

Antes de iniciar cada etapa, foi realizado um piloto com a finalidade de se detectar os possíveis problemas operacionais, como: tempo de duração das tarefas, a opinião dos participantes sobre as tarefas, (grau de dificuldade e interesse pelo material proposto), maneira de aplicação das mesmas, o ambiente físico e os equipamentos utilizados.

Todos os procedimentos, com exceção do levantamento de prontuários, foram realizados em uma única sessão com duas horas de duração.

Esta pesquisa foi aprovada pelo Comitê de Ética em Pesquisa da Pontifícia Universidade Católica de Minas gerais, apresentando o número de Registro CEP: CAAE 0170.0.213.000-05.

A análise estatística foi realizada com um software Minitab 15 por meio do teste de hipótese para proporção, de modo a testar a hipótese nula $(\mathrm{HO})$ de igualdade de proporções. Caso ocorresse valor $p$ menor que o nível de significância determinado a priori de $5 \%$, rejeitava-se a hipótese de igualdade de proporções para cada resultado do teste auditivo. Nesse sentido, a hipótese alternativa $(\mathrm{H} 1)$ era validada, tornando possível considerar determinado resultado no teste auditivo como característica da população estudada. Vale ressaltar que, apesar de ter sido adotado o nível de significância de 5\%, considerou-se como significância marginal os $p$-valores que por estarem próximos do limite de aceitação, foram considerados como tendência a serem significantes.

Além do teste de hipótese, foi realizada inferência estatística por meio do intervalo de confiança para os testes que apresentavam variáveis quantitativas (GIN, Taborga, fala com ruído branco e PSI com palavras), também ao nível de significância de $5 \%$.

\section{RESULTADOS}

No presente estudo foram mostrados na tabela 1 os resultados dos números de indivíduos com alteração em cada um dos seis testes selecionados para avaliar o processamento auditivo nas crianças com queixa de distúrbio de leitura e escrita, dentre os quais: Fala com Ruído, Fala Filtrada, PSI, SSW, Taborga e GIN, e, na tabela 2 estão demonstrados os valores da media de acertos em porcentagem e o intervalo de confiança para a media, bem como a média dos limiares de gap obtidos no teste GIN em milissegundos para o grupo avaliado.

Com base nos dados mostrados na tabela 1 observa-se que a "alteração" nos testes avaliados foi a ocorrência mais frequente nos indivíduos, exceto para o teste de fala filtrada, em que a maioria dos indivíduos da amostra apresentou resultado normal. Apesar da maioria da amostra ter apresentado alteração na maior parte dos testes, apenas nos testes SSW ( $p=0,000)$ e PSI com palavras $(p=0,018)$ essa frequencia de alteração foi estatisticamente significativa. Vale ressaltar que no TABORGA frequência, a alteração teve significância marginal $(p=0,059)$.

A tabela 2 apresenta os resultados de cada teste considerando as variáveis quantitativas, para o qual foi apresentado o intervalo de confiança para cada teste. Observa-se que os valores do intervalo de confiança para a média dos limiares de gap obtidos por meio do GIN na orelha direita variaram entre 5,69 e 10,1, ao passo que na orelha esquerda, entre 5,12 e 8,73.

O teste SSW, identificou que todos os indivíduos mostraram alteração em pelo menos um aspecto das provas que compõem o teste, sendo considerado assim como alterado. (Tabela 1)

\section{DISCUSSÃO}

Para se discutir os aspectos encontrados na pesquisa, vamos nos referir ao testes de acordo com as habilidades envolvidas nos mesmos.

Nos testes GIN e Taborga, pode-se detectar alterações nas habilidades auditivas de resolução e ordenação temporal ( processamento temporal). Tais habilidades estão envolvidas no reconhecimento dos sons da fala, uma vez que mudanças na 
Tabela 1 - Ocorrência de individuos com alteração ou não por teste auditivo

\begin{tabular}{lcccc}
\hline \multicolumn{1}{c}{ Parâmetros } & & $\begin{array}{c}\text { Frequência } \\
\text { Absoluta }\end{array}$ & $\begin{array}{c}\text { Frequência } \\
\text { Relativa }\end{array}$ & p-value \\
\hline \multirow{2}{*}{ GIN } & Normal & 7 & $46,67 \%$ & 0,696 \\
& Alterado & 8 & $53,33 \%$ & 0,500 \\
\hline TABORGA (acertos) & Normal & 4 & $26,67 \%$ & 0,982 \\
FREQUÊNCIA & Alterado & 11 & $73,33 \%$ & $\mathbf{0 , 0 5 9 \#}$ \\
\hline TABORGA (acertos) & Normal & 5 & $33,33 \%$ & 0,941 \\
DURAÇÃO & Alterado & 10 & $66,67 \%$ & 0,151 \\
\hline \multirow{2}{*}{ SSW } & Normal & 0 & $0,00 \%$ & 1,000 \\
& Alterado & 15 & $100,00 \%$ & $\mathbf{0 , 0 0 0 ^ { * }}$ \\
\hline \multirow{2}{*}{ FALA FILTRADA (acertos) } & Normal & 8 & $53,33 \%$ & 0,500 \\
& Alterado & 7 & $46,67 \%$ & 0,696 \\
\hline FALA COM RUÍDO BRANCO & Normal & 5 & $33,33 \%$ & 0,941 \\
(acertos) & Alterado & 10 & $66,67 \%$ & 0,151 \\
\hline \multirow{2}{*}{ PSI COM PALAVRAS } & Normal & 3 & $20,00 \%$ & 0,996 \\
& Alterado & 12 & $80,00 \%$ & $\mathbf{0 , 0 1 8}$ \\
\hline
\end{tabular}

Análise realizada por meio do software Minitab 15.

Legenda: GIN - Gap in Noise; SSW - Staggered Spondaic Word test e PSI - Pediatric Speech Intelligibility test.

Tabela 2 - Resultados dos testes considerando o valor da média de acertos de cada um e o intervalo de confiança (IC) para a média

\begin{tabular}{|c|c|c|c|c|}
\hline \multicolumn{3}{|c|}{ Parâmetros } & Média & IC (95\% P/ Média) \\
\hline \multirow{2}{*}{\multicolumn{2}{|c|}{ GIN }} & $\mathrm{OD}(\mathrm{ms})$ & 7,4 & $(5,69279 ; 10,16436)$ \\
\hline & & $\mathrm{OE}(\mathrm{ms})$ & 6,4 & $(5,12442 ; 8,73272)$ \\
\hline \multirow{2}{*}{\multicolumn{2}{|c|}{ TABORGA (acertos) }} & FREQUÊNCIA (\%) & 34 & $(16,2642 ; 51,7358)$ \\
\hline & & DURAÇÃO (\%) & 54 & $(32,1675 ; 75,8325)$ \\
\hline \multirow{2}{*}{\multicolumn{2}{|c|}{ FALA COM RUÍDO BRANCO (acertos) }} & OD (\%) & 62,9 & $(53,7060 ; 72,1607)$ \\
\hline & & OE (\%) & 72 & $(63,1395 ; 80,8605)$ \\
\hline \multirow{4}{*}{ PSI COM PALAVRAS } & \multirow{2}{*}{$\mathrm{F} / \mathrm{F}$} & OD (\%) & $(60,6)$ & $(45,3617 ; 75,9716)$ \\
\hline & & OE (\%) & $(76,6)$ & $(62,9946 ; 90,3387)$ \\
\hline & \multirow{2}{*}{$\mathrm{F} / \mathrm{R}$} & OD (\%) & $(90,6)$ & $(82,9856 ; 98,3477)$ \\
\hline & & OE (\%) & $(87,3)$ & $(79,9430 ; 94,7237)$ \\
\hline
\end{tabular}

Análise realizada por meio do software Minitab 15.

Legenda: GIN - Gap in Noise; PSI - Pediatric Speech Intelligibility test.

$\mathrm{OD}$ - orelha direita OE - orelha esquerda

duração, pausas e velocidade da sílaba fornecem condições para a compreensão, o que poderia ser considerado como preditor de alterações de leitura, escrita e aprendizagem, tornando-se um pré-requisito para um bom desempenho da compreensão da fala e, portanto, para ser um bom falante ${ }^{21}$.

Os falantes de uma língua possuem um elaborado sistema de controle temporal, que governa tanto a duração específica de cada elemento da fala, como o encadeamento entre eles. Este controle depende da maturação neurológica, das características anátomo-fisiológicas dos articuladores da fala e do código lingüístico. Os fatores temporais devem ser respeitados para que a própria unidade da fala, a sílaba, seja passível de análise. O ritmo e a velocidade da fala dizem respeito à agilidade de encadear os diferentes ajustes motores necessários; psicologicamente, relacionam-se à noção de tempo interior e a rapidez mental do falante, sofrendo grande influência dos aspectos psico-emocionais.

Podemos citar o teste de Padrão de Freqüência que apresentou maior número de alterações quando comparado ao de Padrão de Duração, confirmando os dados encontrados na literatura, como mostra 
um estudo que utilizou uma amostra de 60 crianças, obtendo como resultado maior número de alterações encontradas no teste de padrão de frequência no grupo de estudo, com alterações de leitura, do que as encontradas no grupo controle ${ }^{22}$.

Crianças com distúrbios de linguagem podem apresentar déficit no processamento temporal, demonstrado por limitadas habilidades para identificar alguns elementos fonéticos breves apresentados em contextos de fala específicos e por pior desempenho para identificar ou seqüencializar os estímulos acústicos de curta duração apresentados em sucessão rápida. Existe então, a hipótese de que havendo um déficit de processamento temporal, as crianças poderiam distinguir as breves características da fala e reconstruir corretamente as seqüências de estímulos de entrada se elas fossem apresentadas mais devagar. Isso significa que sujeito com distúrbios de linguagem requer processamento temporal mais longo, apresentando dificuldade para discriminar, seqüencializar ou lembrar estímulos breves em uma sucessão rápida. Portanto, sujeitos com distúrbios de linguagem devem ser prejudicados em percepção e produção de contrastes fonológicos caracterizados por breves ou rápidas mudanças acústicas espectrais.

No teste GIN, pôde-se notar que houve um número alto de alterações, e isso pode ser explicado pelo fato de que o processamento temporal é importante para o desenvolvimento da fala e da leitura, e crianças com alterações de fala podem ter dificuldades no processamento auditivo rápido, as quais podem levar à uma ruptura do sistema fonológico que, por sua vez, prejudicaria o aprendizado da fala e da leitura ${ }^{23}$.

Uma opção terapêutica para essas alterações deve envolver tarefas de seqüência lógico-temporal e prosódia ${ }^{24}$.

Por meio dos testes Fala Filtrada, PSI (monossílabos/ruído) e Fala com Ruído, é possível analisar o aspecto de fechamento auditivo, que se refere à capacidade do ouvinte normal em utilizar redundâncias extrínsecas, que se relacionam com o sinal de fala, para preencher as partes ausentes ou distorcidas do sinal auditivo e reconhecer a mensagem completa. A decodificação auditiva refere-se à identificação dos componentes de uma mensagem auditiva. $\mathrm{O}$ fechamento e a decodificação auditiva capacitam o ouvinte a extrapolar a mensagem completa por meio dos componentes individuais. Desta forma, o fechamento auditivo desempenha um importante papel nas atividades cotidianas do ouvinte, pois raramente o ambiente auditivo cotidiano pode ser considerado ideal. Ao invés disto, é necessário lutar continuamente contra ruídos de fundo, dialetos regionais, interlocutores que falam baixo ou com articulação imperfeita e outros fatores que dificultam a compreensão de mensagens auditivas. Os fatores extrínsecos que auxiliam o fechamento auditivo incluem o conhecimento do assunto, a familiaridade com o vocabulário utilizado, o conhecimento dos aspectos fonêmicos da fala e a familiaridade com as regras do idioma, entre outros. Portanto, se estivermos em uma situação na qual o assunto da conversação seja conhecido, o falante esteja usando vocabulário, sintaxe e semântica familiares, e o ambiente seja acusticamente adequado, precisaremos de pouquíssimo esforço para acompanhar a conversação. Por outro lado, se um ou mais desses fatores estiverem ausentes, precisaremos contar com a presença dos outros fatores e com a representação repetida do sinal auditivo dado pelo Sistema Nervoso Auditivo Central (redundância intrínseca), a fim de conseguir o fechamento auditivo ${ }^{25}$.

No presente estudo, apenas o teste Fala Filtrada mostrou maior número de resultados dentro da normalidade se comparados com os casos alterados, e nos demais testes, PSI (monossílabo/ ruído) e Fala com Ruído, o número de casos alterados foi maior. Isso pode estar relacionado com o fato de que um indivíduo com deficiência de processamento do fechamento auditivo exibe uma falha na redundância intrínseca do Sistema Nervoso Auditivo Central, reduzindo ou eliminando a representação repetida do sinal que chega pelas vias auditivas. Portanto, qualquer intercorrência que reduza a redundância extrínseca do sinal auditivo pode interferir na capacidade do indivíduo em conseguir o fechamento auditivo. Em seu nível mais básico, uma deficiência do fechamento auditivo pode interferir na capacidade de decodificar os aspectos fonêmicos de um sinal de fala. De maneira contrária, o ouvinte com uma deficiência na habilidade de fechamento auditivo, talvez não tenha dificuldade de entendimento da fala em um ambiente acústico ideal, contudo, ele pode apresentar maior dificuldade com ruídos de fundo ou com falantes desconhecidos ${ }^{25}$.

Pode-se dizer então, que uma alteração nesta habilidade pode levar o indivíduo a apresentar déficits na compreensão da fala em ambientes ruidosos, levando a um prejuízo na fala e na linguagem. $\mathrm{Na}$ reabilitação desse indivíduo, faz-se necessário o treinamento auditivo utilizando atividades que envolvam fala com ruído, memória e auxílio para audição ${ }^{24}$.

Nos Testes PSI (figura/história) e SSW, analisamos a habilidade auditiva de figura-fundo, que envolve a atenção seletiva.

A atenção seletiva implica em atender a alguma atividade mental em detrimento de outras. Essa 
habilidade é importante para realização de atividades de leitura em um ambiente ruidoso, e ainda na aprendizagem de um conteúdo escolar novo, em uma sala de aula com outros estímulos auditivos competitivos presentes ${ }^{26}$.

Crianças com dificuldades de aprendizagem ou com alterações de processamento das informações auditivas têm maiores dificuldades para compreender a fala na presença de ruído ${ }^{24}$.

Considerando-se que as salas de aula e os lares são ruidosos, podemos inferir que isto aumenta a dificuldade dessas crianças compreenderem a fala nesses ambientes. Se existe maior dificuldade de atenção seletiva nessas crianças, certamente haverá déficit de comunicação, de aprendizagem escolar, de interação com o meio.

De acordo com a literatura ${ }^{27}$ estas crianças têm inabilidade para analisar fonemicamente 0 som, dificuldades quanto à atenção seletiva, figura-fundo e representação de ordem.

Uma pesquisa ${ }^{28}$ realizada comparou o desempenho no teste de fala com ruído em dois grupos, um com distúrbio de aprendizagem e outro sem. 0 resultado apontou como o de pior performance o grupo com queixa de distúrbio de aprendizagem, o que configura a dificuldade dessas crianças em entender a fala em ambientes auditivamente degradados, como nas salas de aula ${ }^{28}$.

Podemos notar grande déficit nessa habilidade dentre a amostra pesquisada, o que pode ser explicado pelo fato de que alterações nesta capacidade podem ter como características: trocas na escrita (grafêmicas e de orientação direita e esquerda), lentidão para aprender, dificuldade na recepção da linguagem, dificuldade na precisão da leitura de fonemas (trocas articulatórias), o que confirma alterações na decodificação. A terapia para esta alteração pode se basear no treino de análise/síntese fonêmica com apoio visual, melhorar o conhecimento dos fonemas e melhorar o sinal acústico ${ }^{24}$.

Os testes Padrão de Duração e Frequência (Taborga) bem como a presença de inversões no SSW estão relacionados com a habilidade de ordenação temporal e são utilizados para analisar aspectos prosódicos da fala como ritmo, acentuação e entoação ${ }^{20}$.

Um estudo ${ }^{23} \mathrm{com}$ crianças que apresentavam distúrbios de leitura e escrita constatou que essa população apresenta pior desempenho se comparado a crianças sem distúrbios de leitura e escrita, ao analisarem ordenação temporal para estímulos de curta duração. Essa dificuldade de análise poderia ser a explicação comum para os distúrbios de processamento de sons lingüísticos, que na maioria das vezes é composto por estímulos auditivos de curta duração, como, por exemplo, as emissões dos fonemas plosivos. Esse déficit perceptual básico sinaliza uma possível origem para as alterações das representações fonológicas nas crianças portadoras de distúrbios de leitura e escrita.

Em uma pesquisa ${ }^{21}$ crianças com distúrbio específico de leitura, crianças com distúrbio de leitura e atraso de linguagem foram avaliadas e comparadas com um grupo de leitores normais, com idades entre 7 e 10 anos, quanto ao processamento auditivo temporal (ordenação temporal) e decodificação fonologia (leitura de não palavras). As crianças com distúrbio de leitura e atraso de linguagem apresentaram um déficit na ordenação temporal comparada aos leitores normais ${ }^{21}$.

$\mathrm{Na}$ presente pesquisa, foram encontradas alterações em grande parte das crianças, sendo que essas apresentaram alterações no padrão tipo A no teste SSW. Segundo a literatura, nessas crianças, pode haver alterações na conexão temporal, parietal e occipital, influenciando assim na leitura e soletração. A criança geralmente apresenta dificuldade em seqüencialização, organização, soletração, trocas visuais na escrita e pouco capricho ${ }^{24}$.

Assim, pode-se observar que o processo terapêutico para transtornos de leitura e escrita devem incluir de maneira sistemática, exercícios que trabalham a resolução temporal, presença de fala (fonema, sílaba, palavra) e no ruído

Por isso, se faz necessário a avaliação das habilidades do processamento auditivo para a identificação de alterações nas habilidades auditivas e intervenção precoce de crianças com dificuldades escolares ${ }^{29,30}$.

A análise dos resultados demonstra existir uma estreita e estrita relação entre as habilidades do processamento auditivo e o distúrbio de aprendizagem tendo em vista a importância de se determinar a existência do comprometimento das habilidades auditivas em crianças com transtornos de aprendizagem, uma vez que esta informação poderá nortear as estratégias do tratamento e indicar as metas educacionais.

Porém, com base neste estudo nota-se ser necessária a análise de uma amostra mais ampla a fim de conceder maior credibilidade às hipóteses levantadas.

\section{CONCLUSÃO}

Verificou-se estreita e estrita relação entre as habilidades do processamento auditivo e o distúrbio de leitura e escrita. Os procedimentos que se destacaram na identificação de anormalidades no processamento neurológico da informação auditiva foram os testes SSW, PSI com palavras no ruído e Teste de Padrão de Freqüência melódico. 


\section{ABSTRACT}

Purpose: to check the incidence of possible disorders in children with reading and writing auditory processing disorder. Method: we selected 15 children with normal hearing, in the age group of 8 to 12 years, with low school income and that were being treated in the sector of written language in the Clinical Center of Speech and Hearing of Pontifical University Catholic of Minas Gerais. The individuals were submitted to the following tests: Pediatric Speech Intelligibility test, Pediatric Speech Intelligibility test, Speech with noise, Speech Filtered, Gap in Noise and Standard of Duration and Frequency. Results: it was found that all children had changes in at least one hearing ability in auditory processing. Conclusions: there was strict and close relationship between the auditory processing skills and reading and writing disorder. The procedures which excelled in identifying neurological abnormalities in auditory information processing tests were SSW, PSI with words in noise and Test Frequency Pattern melodic.

KEYWORDS: Language; Underachievement; Hearing; Child; Reading

\section{REFERÊNCIAS}

1. Simon LF, Rossi AG.Triagem do processamento auditivo em escolares de 8 a 10 anos. Psicol. esc. Educ .2006;10(2):293-304.

2. Nunes C, Frota S, Mousinho R. Consciência fonológica e o processo de aprendizagem de leitura e escrita: implicações teóricas para o embasamento da prática fonoaudiológica. Cefac. 2009;11(2):207-12.

3. Lima TCF, Pessoa ACR. Dificuldade de aprendizagem: principais abordagens terapêuticas discutidas em artigos publicados nas principais revistas indexadas no LILACS de fonoaudiologia no período de 2001 a 2005. Cefac. 2007; 9(4):469-76. 4. Nicolielo AP, Fernandes GB, Garcia VL, Hage. SRV. Desempenho escolar de crianças com Distúrbio Específico de Linguagem: relações com habilidades metafonológicas e memória de curto prazo. Soc. bras. fonoaudiol. 2008; 13(3):246-50.

5. Navas ALG, Pinto JCB, Dellisa PRR. Avanços no conhecimento do processamento da fluência em leitura: da palavra ao texto. Soc. bras. fonoaudiol. 2009;14(4):553-9.

6. Murphy CFB, Schochat E. Correlações entre leitura, consciência fonológica e processamento temporal auditivo. Pró-Fono R. Atual. Cient. 2009;21(1):13-8.

7. Abdo AGR, Murphy CFB, Schochat E. Habilidades auditivas em crianças com dislexia e transtorno do déficit de atenção e hiperatividade. Pró-Fono R. Atual. Cient. 2010;22(1):25-30.

8. Santos JN, Lemos SMA, Rates SPM, Lamounier JA. Habilidades auditivas e desenvolvimento de linguagem em crianças. Pró-Fono R. Atual. Cient. 2008; 20(4):255-60.
9. Andrade NA, Gil D, Schiefer AM, Pereira LD. Processamento auditivo em gagos: análise do desempenho das orelhas direita e esquerda. Soc. bras. fonoaudiol. 2008;13(1):20-9.

10. Lucas PA. Scan: perfil de desempenho em crianças de sete e oito anos. Pró-Fono. 2007;19(4):370-3.

11. Zalcman TE, Schochat E. A eficácia do treinamento auditivo formal em indivíduos com transtorno de processamento auditivo. Soc. bras. fonoaudiol. 2007; 12(4): 310-14.

12. Balen AS, Massignani R, Schillo R. Aplicabilidade do software Fast Forword na reabilitação dos distúrbios do processamento auditivo: resultados iniciais. Rev. CEFAC. 2008;10(4):572-87.

13. Deuschle V P, Cechella $C$. O déficit em consciência fonológica e sua relação com a dislexia: diagnóstico e intervenção. Rev. CEFAC. 2009; 11(2): 194-200.

14. Tiensoli LO, Goulart LMH, Resende LM, Colosimo EA. Triagem auditiva em hospital público de Belo Horizonte, Minas Gerais, Brasil: deficiência auditiva e seus fatores de risco em neonatos e lactentes. Cad. Saúde Pública. 2007; 23(6):1431-41. 15. Pereira LD, Schochat E. Processamento auditivo central: Manual de avaliação. São Paulo: Lovise; 1997.

16. Lemos ICC. Sistema de freqüência modulada no transtorno do processamento auditivo: prática baseada em evidências?. Pró-Fono R. Atual. Cient. 2009;21(3):243-8.

17. Garcia VL, Campos DBKP, Padovani CR. Associação entre a avaliação de habilidades de consciência fonológica e de processamento auditivo em crianças com e sem distúrbio de aprendizagem. Fono Atual. 2005; 8(31): 4-11. 
18. Northern JL, Downs MP. Development of auditory behavior: hearing in children. 5th ed. Baltimore: Williams and Wilkins. 2002.

19. Musiek FE, Zaidan EP, Baran JÁ, Shinn JB, Jirsa RE. Assessing temporal processes in adults with LD: the GIN test. In: Convention of American Academy of Audiology. 2004 march/april; Salt Lake City. Annals. Salt Lake City: AAA, pp203, 2004.

20. Taborga MBL. Processos temporais auditivos em músicos de Petrópolis [Monografia]. São Paulo: Universidade Federal de São Paulo. Escola Paulista de Medicina; 1999.

21. Ishi C, Arashiro PM, Pereira LD. Ordenação e resolução temporal em cantores profissionais e amadores afinados e desafinados. Pró-Fono $\mathrm{R}$. Atual. Cient.2006 Set/Dez; 18(3):285-92.

22. Murphy CFB, Schochat E. Correlações entre leitura, consciência fonológica e processamento temporal auditivo. Pró-Fono R. Atual. Cient. 2009;21(1)13-8

23. Tallal $\mathrm{P}$, Miller $\mathrm{S}$, Fitch $\mathrm{RH}$. Neurobiological basis of speech: a case for the preeminence of temporal processing. Annals of the New York Academy of Sciences. 1993;682:27-47.
24. Katz J. Classification of auditory processing disorders. In: Katz J, Stecker NA, Handerson D. ed. Central auditory processing: a transdiciplinary view. St. Louis, Mosby Yearbook. 1992;81-91.

25. Jacob LCB, Alvarenga KF, Zeigelboim BS. Avaliação audiológica do sistema nervoso auditivo central. Arquivos da Fundação de Otorrinolaringologia. 2000 Out/Dez; 4(4):144-51.

26. Garcia VL, Pereira LD, Fukuda Y. Atenção seletiva: PSI em crianças com distúrbio de aprendizagem. Rev. Bras. Otorrinolaringol. 2007 Mai/Jun; 73(3): 404-11.

27. Vitto LPM. Macrossomia e habilidades auditivas: estudo comparativo. Pró-Fono R. Atual. Cient. 2005;17(2):223-32.

28. Santos FS, Schochat E. Dificuldade de ouvir na presença de ruído e dificuldade de aprendizagem. Rev Fonoaudiologia Brasil. 2003;2(3):36-42.

29. Neves IF, Schochat E. Maturação do processamento auditivo em crianças com e sem dificuldades escolares. Pró-Fono R. Atual. Cient. 2005; 17(3):311-20.

30. Zalcman TE, Schochat E. A eficácia do treinamento auditivo formal em indivíduos com transtorno de processamento auditivo. Soc. Bras. Fonoaudiol. 2007;12(4):310-4.

doi: 10.1590/S1516-18462010005000119

RECEBIDO EM: 27/01/2010

ACEITO EM: 24/07/2010

Endereço para correspondência:

Karen Maria de Paula

Rua Furquim, 473, Pompéia

Belo Horizonte - MG

CEP: 30280-490

E-mail: karen_paula2000@yahoo.com.br 